\title{
AVALIAÇÃO DO RISCO DE EXTRAVASAMENTO DE QUIMIOTERÁPICO ANTINEOPLÁSICO ADMINISTRADO VIA CATETER DE INSERÇÃO PERIFÉRICA: RELATO DE CASO
}

\author{
Walterson RADAEL ${ }^{1}$, Wanderson RADAEL ${ }^{1}$, Arandir CARVALHO ${ }^{1}$, Josileyde DUTRA ${ }^{1}$, Bianca \\ LAZARONE $^{2}$ \& Luciana FUMIAN ${ }^{*}$
}

\footnotetext{
${ }^{1}$ Universidade Iguaçu - Campus V, Itaperuna, Rio de Janeiro, Brasil.

${ }^{2}$ Hospital São José do Avaí, Itaperuna, Rio de Janeiro, Brasil.

*Autor para correspondência: lucianafumian@gmail.com
}

http://dx.doi.org/10.18571/acbm.104

\section{RESUMO}

O extravasamento de quimioterápicos antineoplásicos se destaca como sendo a complicação aguda mais severa entre os eventos relacionados à administração desses fármacos por via intravenosa. Objetivo: relatar o caso de uma paciente com 49 anos de idade, que desenvolveu lesão cutânea devido ao extravasamento de droga quimioterápica administrada via cateter de inserção periférica. Materiais e Métodos: este trabalho constitui-se de um relato de caso de uma paciente diagnosticada com câncer de mama, que ao decorrer do tratamento sobreveio o extravasamento de droga antineoplásica. Tendo a equipe de saúde adotado todos os protocolos e condutas necessárias a fim de minimizar os agravos e tratar a lesão. Resultados: foi possível evidenciar a remissão completa da lesão após um período aproximado de quatro semanas após o ocorrido. Conclusão: a composição qualificada da equipe de profissionais da saúde, assim como a padronização dos protocolos relacionados ao extravasamento de antineoplásicos, visam reduzir os riscos de lesões e aumentam a qualidade de vida do paciente.

Palavras-chave: Extravasamento de Antineoplásico; Cateter de Inserção Periférica; Protocolos.

\begin{abstract}
The extravasation of anticancer chemotherapeutic stands out as being the most severe complication of acute events related to the administration of these drugs intravenously. Objective: To report the case of a patient with 49-year-old woman who developed skin lesion due to the chemotherapy drug extravasation administered via peripherally inserted catheter. Materials and Methods: This work constitutes a case report of a patient diagnosed with breast cancer, that the course of treatment came antineoplastic drug extravasation. With the health team adopted all protocols and behaviors necessary in order to minimize injuries and treat injury. Results: it was possible to demonstrate the complete remission of the lesion after a period of approximately four weeks after the incident. Conclusion: The composition of the qualified health professional staff, as well as standardization of protocols related to extravasation of anticancer, aim to reduce the risk of injury and increase the quality of life of patients.
\end{abstract}

Keywords: Antineoplastic Extravasation; Peripherally Inserted Catheter; Protocols. 


\section{Introdução}

O termo câncer é derivado do latim câncer e do grego karkínos (caranguejo), e engloba mais de duzentos tipos distintos da doença, cuja característica principal é marcada pela ocorrência de alterações nos processos de divisão celular do corpo humano. Essas desordens proporcionam um crescimento anormal de um grupo de células dando origem ao que genericamente se denomina de tumor (FRANKS \& TEICH, 1990).

Segundo dados da Organização Mundial da Saúde, houve um total de 14,1 milhões casos novos de câncer e um total de 8,2 milhões de mortes por câncer em todo o mundo em 2012. Estima-se que em 2030, essa carga global seja de, aproximadamente, 21,4 milhões de casos incidentes e 13,2 milhões de óbitos, em virtude do envelhecimento e aumento da densidade populacional, bem como da redução da mortalidade infantil e nos falecimentos por doenças infecciosas nos países em desenvolvimento (INCA, 2014).

O câncer de mama é o tipo neoplasia que mais acomete as mulheres em todo o mundo, tanto em países subdesenvolvidos quanto em países desenvolvidos. Na população global, foram esperados para o ano de 2012 cerca de 1,67 milhões de casos novos desse tipo de tumor, o que representa $25 \%$ de todos os tipos de câncer diagnosticados no gênero feminino. Suas taxas de incidência variam entre as diferentes regiões do mundo, apresentando taxas mais elevadas na Europa Ocidental (96/ 100 mil) e as menos elevadas na África Central e na Ásia Oriental (27/ 100 mil) (INCA, 2014).

No tratamento do câncer, a via intravenosa é a mais utilizada para a administração de quimioterápicos por garantir uma rápida absorção e um nível sérico adequados para os mais variados grupos farmacológicos disponíveis. Dentre os principais eventos adversos relacionados à administração intravenosa, o extravasamento se destaca como a complicação aguda mais severa, causando extremo desconforto e sofrimento ao paciente e exigindo do enfermeiro habilidade clínica para diagnosticá-lo e intervir precocemente, para que seja adotado protocolos padronizados com finalidade de melhorar a qualidade de vida do paciente oncológico (CHANES, et al, 2008).

O extravasamento dos antineoplásicos é definido, como o escape das drogas do vaso sanguíneo para os tecidos circunjacentes, e seus efeitos tóxicos locais variam podendo causar dor, necrose tissular, formação de vesículas, edema, queimação, desconforto local e eritema. Os quimioterápicos vesicantes são os responsáveis pelas reações mais graves, pois geram irritação severa com formação de vesículas e destruição tecidual quando infiltrados fora do vaso sanguíneo. Já os quimioterápicos classificados como irritantes, quando extravasados, provocam reação cutânea menos intensa, como dor e queimação, sem necrose tecidual ou formação de vesículas (ADAMI, et al, 2001).

A incidência de extravasamento de drogas vesicantes é de $0,5 \%$ a $6,4 \%$ em pacientes que recebem a quimioterapia através de cateter de inserção periférica. Há fatores que contribuem para o aumento no risco de extravasamento como o uso de veias pequenas e frágeis, erros técnicos na punção venosa periférica e na administração das drogas aplicadas ao tratamento (CORREIA, et al, 2011).

\section{Materiais e Métodos}

A metodologia apresentada refere-se ao relato de caso de uma paciente adulta, com 49 anos de idade, inicialmente diagnosticada com Fibroadenoma Intracanalicular, tendo evolução do quadro clínico para um Carcinoma Ductal Invasivo.

Após vários ciclos de protocolos de tratamento, que teve início em outubro de 2012, a paciente começou apresentar sintomas característicos de extravasamento de quimioterápico antineoplásico, no qual estava sendo administrado via cateter de inserção periférica. Prontamente 
a equipe de enfermagem interrompeu o tratamento e adotou as condutas necessárias a fim de minimizar agravos.

Tendo adotado a aplicação de compressas mornas na região afetada, adequadas ao extravasamento de um alcaloide da vinca. Dando sequência ao tratamento foi aplicado um antiinflamatório esteroidal por via tópica, sendo que após o aparecimento total das lesões foi prescrito um anti-inflamatório não-esteroidal para ser administrado concomitante por via oral.

Este relato de caso foi autorizado pela paciente, sendo que foi assinado o termo de consentimento livre e esclarecido, o qual permite o uso da imagem e o relato do caso para publicação, seguindo, portando, os preceitos éticos da resolução 466/12 do Conselho Nacional de Saúde.

\section{Relato de Caso}

Paciente E.S.A.S, $1^{\circ}$ grau incompleto, 49 anos, natural de Itaperuna, reside na zona rural, profissão costureira. Histórico familiar com pai que teve câncer em região torácica.

Nega Hipertensão, diabetes ou algum tipo de alergia a medicamentos. Não utiliza hormônio feminino. Menarca aos 17 anos.

Paciente foi submetida a exame devido a nódulo em Mama direita, sendo diagnosticada com Fibroadenoma Intracanalicular. Desde então mantém controle com a realização de exames periódicos para acompanhamento da evolução.

Após algum tempo, relatou início de dor no ombro direito. Submetida a novos exames, fora diagnosticado nódulo espiculado de margens irregulares, sugestivo de carcinoma ductal invasivo.

Iniciou a primeira sessão de quimioterapia no dia 16/10/2012, com esquema FAC (Fluorouracila + Doxorrubicina + Genuxal). Depois disso, necessária foi à mudança de protocolo passando para o tratamento com Taxano (Paclitaxel). Após resultado do teste de Hibridização Fluorescente in Situ (Fish) positivo, passou para quimioterapia adjuvante com Herceptin + Taxano. Finalizado esse ciclo, iniciou-se o protocolo Gencitabina + Vinorelbine, ambos administrados por via endovenosa. Observou-se que posteriormente à primeira administração de Navelbine endovenoso em bolus a paciente começou a queixar-se de dor e queimação em membro superior esquerdo, com início de edema na região.

Imediatamente, a equipe de enfermagem interrompeu a administração da medicação e adotou os procedimentos para extravasamento de quimioterápicos antineoplásicos como: interrupção da administração do medicamento, aspiração da droga no local do acesso por meio de seringa, retirada do acesso venoso, aplicação compressas mornas no local da infusão, notificação ao setor de farmácia e aplicação de corticoide tópico no local do extravasamento.

Paciente foi encaminhada à avaliação médica que orientou continuação da conduta em domicílio, com aplicação de bolsas mornas de três a quatro vezes ao dia e aplicação de corticoide tópico durante cinco dias, agendando retorno semanal ao setor de oncologia para avaliação da derme pela equipe de enfermagem.

Transcorridos três dias após o extravasamento do fármaco apareceram as lesões. Paciente retornou ao serviço de oncologia (figura 1) e foi orientada a manter a conduta adotada e a iniciar Nimesulida $100 \mathrm{mg}$ de 6/6 horas durante cinco dias por via oral. Houve remissão completa da lesão após aproximadamente quatro semanas de tratamento (figura 2).

Em razão do ocorrido a equipe médica optou em fazer a substituição do Vinorelbine endovenoso pelo Vinorelbine oral. 


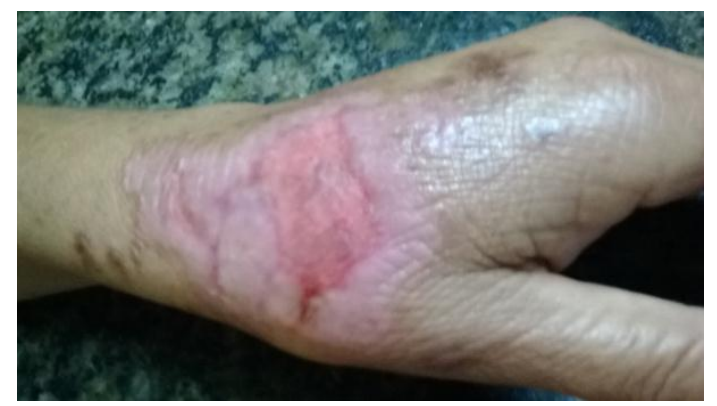

Figura 1: Lesão após três dias de extravasamento do fármaco.

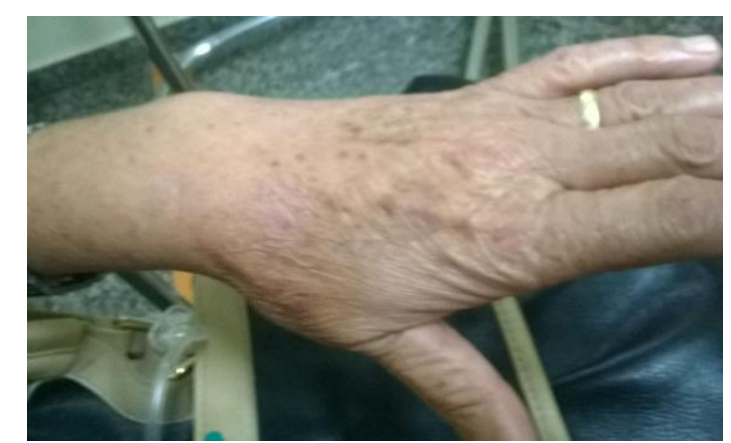

Figura 2: Remissão da lesão após, aproximadamente, quatro semanas de tratamento.

\section{Resultados}

Os fatos evidenciaram a completa remissão da lesão, tendo a integridade da derme reestabelecida, demonstrando eficiência nos métodos adotados pela equipe assistencial do setor de oncologia onde a paciente se encontra em tratamento, em prol da resolução do dano causado pelo extravasamento do quimioterápico antineoplásico.

Imediatamente após a infusão da medicação a paciente relatou queixas de dor e ardor no sitio da infusão. Iniciado protocolo para extravasamento de quimioterápico e orientado pela equipe de enfermagem para manutenção em domicílio até completa involução da ferida e agendado novo retorno para avaliação da derme. O aparecimento das lesões se deu com aproximadamente três dias após o ocorrido, retornando a paciente ao setor de oncologia onde a equipe médica manteve as condutas adotadas e orientou a administração oral de nimesulida 100 mg na frequência de 6/6 horas durante cinco dias.

Após cerca de quatro semanas de tratamento a paciente apresentou remissão total da ferida e não houve mais relatos de dor ou queimação no sítio da lesão.

Por conseguinte, verificamos a importância da atuação multiprofissional a favor do tratamento e da manutenção da qualidade dos pacientes assistidos. Foi dado sequência ao tratamento quimioterápico, porém houve uma alteração quanto à forma de administração, substituindo a endovenosa para a quimioterapia oral.

\section{Discussão}

A morbidade está diretamente relacionada à quantidade da droga extravasada, à sua concentração, à localização, ao tipo de dispositivo usado no acesso venoso, às condições de saúde do paciente e ao intervalo entre a ocorrência do fato, seu reconhecimento e o início do tratamento (GOZZO, et al, 2010).

As drogas vesicantes são aquelas que, quando ocorre o extravasamento, provocam irritação severa, com formação de vesículas e necrose tecidual, podendo ainda causar danos funcionais, alterações neurológicas, alterações na imagem corporal e, podendo levar a perda da confiança do 
paciente. Entretanto, as drogas irritantes, provocam reações cutâneas de menor intensidade, como dor e queimação, contudo, mesmo quando administradas adequadamente, podem causar dor e reação inflamatória ao longo do vaso sanguíneo empregado na infusão (GOZZO, et al, 2010).

Após a identificação do extravasamento, é preciso tomar condutas para que os danos ao paciente sejam reduzidos, como: interromper a infusão como primeiro cuidado, manter a agulha no local e aspirar à droga antineoplásica, aplicação de compressas no local. Além disso, se faz importante, fotografar o local do extravasamento, registrar o ocorrido e estabelecer um plano de acompanhamento e cuidados (SCHNEIDER e PEDROLO, 2011).

O tempo de infusão e o fluxo prescrito são fatores relevantes na monitorização dos quimioterápicos antineoplásicos. Além do mais, dispositivos com menor calibre diminuem o trauma nos vasos durante o procedimento da punção e, sendo menor que o do vaso facilita a circulação de sangue ao redor do dispositivo, auxiliando na hemodiluição das drogas administradas (RODRIGUES, et al, 2012).

Em decorrência das alterações anatômicas das veias e do risco de complicações em pacientes que fazem uso de antineoplásicos vesicantes, a ordem de punção das veias difere da ordem normalmente utilizada. Deve-se iniciar pelo antebraço, logo, passar para o dorso das mãos, punho e fossa antecubital, sendo que para cada local escolhido a punção venosa deve ser realizada seguindo ao sentido distal-proximal (SCHNEIDER e PEDROLO, 2011).

Quanto ao paciente, é importante que este seja devidamente orientado, quanto aos riscos de complicações envolvidas na administração de drogas vesicantes através de infusão endovenosa periférica, para que o mesmo relate qualquer sintoma de dor, desconforto e queimação na região em que o cateter foi inserido. Caso o profissional de saúde envolvido não consiga distinguir se o sintoma possa ser indício de extravasamento, deve interromper imediatamente a infusão e verificar se o cateter está adequadamente inserido dentro do vaso, observando o aparecimento de lesões circunjacentes (FERREIRA, et al, 2008).

A educação de pacientes e cuidadores são fundamentais, sendo que as orientações verbais ao paciente oncológico devem também vir seguidas de informações escritas, como forma de orientação de como proceder em caso de aparecimento de complicações no local de administração da droga (FERREIRA, et al, 2008).

\section{Conclusão}

Tendo em vista os aspectos abordados, é de extrema importância a composição qualificada da equipe de profissionais da saúde, envolvidos em todo o processo da terapia com drogas quimioterápicas antineoplásicas.

Assim sendo, os protocolos relacionados ao extravasamento de antineoplásicos devem ser padronizados, buscando reduzir os riscos de lesões e aumentando a qualidade de vida do paciente.

\section{Referências}

ADAMI, Nilce Piva; et al. Extravasamento de Drogas Antineoplásicas Notificação e Cuidados Prestados. Revista Brasileira de Cancerologia, São Paulo, v. 47 (2): 143-51; 2001.

CHANES, Daniella Cristina; DIAS, Carla Gonçalves; GUTIÉRREZ, Maria Gaby Rivero. Extravasamento de Drogas Antineoplásicas em Pediatria: Algoritmos para Prevenção, Tratamento e Seguimento. Revista Brasileira de Cancerologia, São Paulo, v. 54 (3): 263-273; 2008. 
CORREIA, Jefferson Nery; ALBACH, Letiery Sanches Pereira; ALBACH, Carlos Augusto. Extravasamento de quimioterápicos: conhecimentos da equipe de enfermagem. Revista Ciência \& Saúde, Porto Alegre, v. 4 (1): 22-31; 2011. Semestral.

FERREIRA, Marizete Tavares; REIS, Paula Elaine Diniz dos; GOMES, Isabelle Pimentel. Prevenção de Extravasamento por Quimioterapia Antineoplásica. OBJN, Brasília, v. 7 (3); 2008.

FRANKS, L.M. \& TEICH, N. Introdução à Biologia Celular e Molecular do Câncer. São Paulo: Livraria Roca Ltda, 1990.

GOZZO, Thais de Oliveira; et al. Toxicidade dermatológica em mulheres com câncer de mama submetidas à quimioterapia. Revista Latino-Americana de Enfermagem, Ribeirão Preto, v. 18(4): 07 telas; 2010. Mensal.

INCA Incidência de Câncer no Brasil: Câncer da mama feminina. 2014. Disponível em: http://www.inca.gov.br/estimativa/2014/sintese-de-resultados-comentarios.asp. Acesso em: 17/10/2015.

RODRIGUES, Cíntia Capucho; et al. Fatores de risco para trauma vascular durante a quimioterapia antineoplásica: contribuições do emprego do risco relativo. Revista Acta Paulista de Enfermagem, Ribeirão Preto, v. 25 (3): 448-52; 2012.

SCHNEIDER, Franciane; PEDROLO, Edivane. Extravasamento de Drogas Antineoplásicas: Avaliação do Conhecimento da Equipe de Enfermagem. Revista Mineira de Enfermagem, Curitiba, v. 15 (4): 522-529; 2011. Bimestral. 